\title{
Prevalence of Anti-Cardiolipin and Anti- $\beta 2$ Glycoprotein Antibodies in Indian Systemic Lupus Erythematosus Patients
}

\author{
Vandana Pradhan', Anjali Rajadhyaksha ${ }^{1}$, Pranaya Joshi ${ }^{1}$, Manisha Patwardhan ${ }^{1}$, Shruti Dighe ${ }^{1}$, \\ Kanjaksha Ghosh ${ }^{2}$
}

\footnotetext{
${ }^{1}$ Department of Autoimmune Disorders, National Institute of Immunohaematology, Indian Council of Medical Research, King Edward Memorial Hospital, Mumbai, India. ${ }^{2}$ Department of Medicine, King Edward Memorial Hospital, Mumbai, India.

Email: pradhanv69@rediffmail.com
}

Received March 15 ${ }^{\text {th }}, 2011$; revised April 26 ${ }^{\text {th }}, 2011$; accepted May $27^{\text {th }}, 2011$.

\begin{abstract}
Anti-phospholipid antibodies (APA) like anti-cardiolipin antibodies $(A C A)$ and anti- $\beta 2$ glycoprotien (anti- $\beta 2$ GP) are important cause of venous and arterial thrombosis and other occlusive vascular diseases. The prevalence of these antibodies in SLE patients at the time of diagnosis is not known in Indian SLE patients. This study was conducted to evaluate the prevalence of ACA and anti- $\beta 2$ GP autoantibodies in SLE patients and to correlate them with disease activity and immune parameters such as C3,C4 and CRP levels, where 85 SLE patients referred from Rheumatology Department, KEM hospital, Mumbai were studied. SLE disease activity was evaluated by SLE Disease Activity Index (SLEDAI) score at the time of evaluation. All patients studied were in an active stage of disease of which $37.6 \%$ patients had renal disorders, which were categorized as Lupus Nephritis $(L N)$ and $62.3 \%$ patients did not show any renal manifestations (non-LN). ACA and anti- $\beta 2$ GP autoantibodies, to IgG and IgM subclasses were tested by ELISA. C3, C4 and CRP levels were detected by nephelometer. It was observed that $12.9 \%$ patients were IgG-ACA and IgM-ACA positive and ACA positivity was noted more among $L N$ group Anti- $\beta 2$ GP autoantibody positivity was $27.1 \%$ for $\operatorname{IgG}$ and $31.8 \%$ for IgM., IgG-anti- $\beta 2$ GP antibodies were slightly higher in non-LN patients, whereas a higher incidence of IgM-anti- $\beta 2$ GP antibodies were detected in LN patients. Hence detection both ACA and anti- $\beta 2$ GP antibodies along with associated immune parameters were helpful to evaluate their possible association with disease severity in SLE patients. A long term follow up of patients having ACA and anti- $\beta 2$ GP antibodies without thrombotic event is also needed to detect their possible thrombotic event in future along with their clinical presentation.
\end{abstract}

Keywords: Systemic Lupus Erythematosus (SLE), Anti-Cardiolipin Antibodies (ACA), Anti- $\beta 2$ Glycoprotein Antibodies (anti- $\beta 2$ GP), Lupus Nephritis (LN), SLE without Nephritis (Non-LN)

\section{Introduction}

Antiphospholipid antibody syndrome (APS) is perhaps one of the most confounding immunologic disorders. It's an acquired autoimmune disorder defined by the presence of antibodies against phospholipids. Anti-phospholipid antibodies (APA), namely the lupus anticoagulant (LAC) and the anti-cardiolipin antibodies (ACA) are a group of antibodies directed against negatively-charged phospholipid antigens (phosphatidylserine), on endothelial cell membranes and platelets. Previously these antibodies were thought to be recognizing epitopes on anionic phospholipids and a complex of lipid-bound human prothrombin [1]. Several components including high titres of APA, $\beta 2$ GP-I and activation of endothelium or platelets, are now suspected to encompass the APS [2]. It seems likely that binding of APA on endothelial cells is mediated through the cofactor $\beta 2$ GP-I [3]. The target antigen for could be a complex of $\beta 2$ GP-I and anionic phosphoator lipids.

Systemic Lupus Erythematosus (SLE) is a chronic, multisystem, inflammatory disorder of autoimmune etiology, occurring predominantly in young women in their child-bearing age. Common manifestations may include arthralgias and arthritis, malar and other skin rashes, pleuritis or pericarditis, renal and CNS involvement and hematologic cytopenias. SLE may be precipitated by currently unknown environmental triggers that cause autoimmune reactions in genetically predisposed people $[4,5]$. Lupus Nephritis (LN) is histologically evident in 
most patients with SLE with the involvement of varying degree of renal disease. Autoimmunity plays a major role in the pathogenesis of $\mathrm{LN}$ where autoantibodies form pathogenic immune complexes that deposit in kidneys. Glomerular thrombosis is another mechanism that may play a role in pathogenesis of $\mathrm{LN}$, mainly in patients with APS and is believed to be the result of antibodies directed against negatively charged phospholipid-protein complexes [6,7]. APS is classified as primary or secondary depending on its association with other autoimmune disorders. Primary APS is diagnosed in patients demonstrating the clinical and laboratory criteria without other recognized autoimmune disease. Secondary APS is diagnosed in patients with other autoimmune disorders such as SLE. One-third of patients with SLE also have antiphospholipid antibodies, and approximately one-third of those with antibodies have clinical signs of antiphospholipid antibody syndrome [8].

Phospholipids such as cardiolipin, $\beta 2$ glycoprotein and LAC are responsible for prevention of blood clotting. In patients with SLE who have bad obstetric history (BOH) or recurrent pregnancy loss (RPL), both cardiolipin and lupus anticoagulant antibodies are often present in high titre [9]. ACA may belong to both IgG and IgM subtypes. The IgG antibodies seem to be better predictors of fetal outcome. More recent studies suggest that the antibodies that really matter are those to $\beta 2 \mathrm{GP}$, the cofactor by which ACA binds to phospholipid and usually are present with ACA [10]. Earlier studies have confirmed that patients' positive for ACA are at risk of repeated episodes of thrombosis, fetal loss and thrombocytopenia. APA occurs in up to $60 \%$ of patients with SLE and may be of pathogenic significance in $\mathrm{LN}$ where the presence of intra glomerular capillary thrombosis has also been described [11-14].

Present study was designed to evaluate the prevalence of ACA and anti- $\beta 2$ GP autoantibodies in SLE patients and to correlate them with disease activity and immune parameters.

\section{Materials and Methods}

This study was conducted in 85 SLE patients from Rheumatology department of KEM hospital, Mumbai, India over the period of 2 years (2008-2010). SLE patients were diagnosed according to the American College of Rheumatology (ACR) criteria [15]. This study was carried out after obtaining the requisite Ethics Committee approval and a written consent from the patient. The disease activity was assessed at the time of evaluation using the Systemic Lupus Erythematosus Disease Activity Index (SLEDAI) [16]. Pregnant and post menapausal women, smokers, patients with diabetes and patients with significant hyperlipemia were excluded. After blood col- lection, sera were stored in aliquots at $-80^{\circ} \mathrm{C}$ until tested. Renal biopsies of Lupus Nephritis (LN) cases were examined by light microscopy using hematoxylin, eosin, periodic Schiff (PAS) staining. Immunofluorescence microscopy was done using anti-IgG, anti-IgM, anti-IgA, anti-C3, anti-C4 and anti-fibrinogen fluorescein isothiocyanate conjugate (FITC). In LN patients the renal histology was classified according to WHO criteria [17]. Anti-Cardiolipin antibodies (ACA) to IgG and IgM isotypes and anti- $\beta 2$ GP autoantibodies to $\operatorname{IgG}$ as well as IgM isotypes were detected by ELISA using commercially available kits (Euroimmune, Lubeck). C3, C4 and CRP levels were detected using a Nephelometer (BN ProSpec, Dade Behring, Germany). The Laboratory was blinded to the disease status of patients and their visceral involvement and a double blinded study was conducted on the autoantibody positive samples.

\section{Results}

A total 85 SLE patients were included in the study of which 80 were females and remaining 5 were males. The ages ranged between 16 yrs and 36 yrs with mean age being $26.8 \pm 9.9$ yrs. According to the ACR criteria, clinical manifestation of patients indicated that majority of these patients $(60 \%)$ had arthritis followed by rash (Malar and Discoid) among 38.8\% patients. It was observed that renal disorders were seen among 32/85 (37.6\%) patients and these patients were categorized as LN. Remaining 53 patients $(62.4 \%)$ did not showed renal manifestation and was categorized as non-LN. Other manifestations such as photosensitivity $(20 \%)$, serositis (24.7\%) and neurological disorder (16.5\%) were noted among the study group. Laboratory findings indicated that all these patients were in active stage of disease where ANA positivity was noted among all. Hematological disorders showed anemia $(\mathrm{Hb} \leq 7.0 \mathrm{~g} / \mathrm{dl})$ and/or autoimmune hemolytic anemia (AIHA) in $27.1 \%$, leucopenia $\left(\mathrm{WBC}\right.$ count $\left.\leq 4.0 \times 10^{3} / \mu \mathrm{l}\right)$ in $16.5 \%$, lymphopenia (total lymphocyte count $\leq 2.0 \times 10^{3} / \mu \mathrm{l}$ ) in $11.8 \%$ and thrombocytopenia (platelet count $\leq 150 \times$ $10^{3} / \mu \mathrm{l}$ ) in $25.9 \%$ patients. Immunological disorders showed raised CRP levels $(>5 \mathrm{mg} / \mathrm{L})$ in $51.8 \%$ patients where $61.4 \%$ patients had previous history of bacteria, viral or parasitic infections and remaining $38.6 \%$ patients had no history of previous infections. Decreased C3 levels alone $(<90-180 \mathrm{mg} / \mu \mathrm{l})$ were detected in $22.4 \%$ patients whereas in $13 \%$ patients, a decreased $\mathrm{C} 4$ levels alone $(<10-40 \mathrm{mg} / \mu \mathrm{l})$ were noted. Both $\mathrm{C} 3$ and $\mathrm{C} 4 \mathrm{lev}-$ els were reduced in $27.1 \%$ patients where as $37.7 \%$ patients had normal C3 as well as C4 levels (Table 1).

As shown in Table 2, it was observed that $12.9 \%$ patients had developed IgG-ACA and IgM-ACA individu ally each where as $3.5 \%$ of patients had developed both 
Table 1. Baseline characteristics of SLE patients according to ACR criteria $(n=85)$.

\begin{tabular}{lc}
\hline \multicolumn{2}{c}{ Characteristics: } \\
\hline $\begin{array}{l}\text { Sex Ratio (F:M) } \\
\text { Mean Age (Years } \pm \text { SD) }\end{array}$ \\
\multicolumn{1}{c}{ Clinical Manifestations (\%) } \\
\hline Rash (Malar or Discoid) & $26.8 \pm 9.9$ \\
Photosensitivity & $17(20.8 \%)$ \\
Oral Ulcers & $24(28.2 \%)$ \\
Arthritis & $51(60 \%)$ \\
Serositis & $21(24.7 \%)$ \\
Renal Disorders & $32(37.6 \%)$ \\
Neurological Disorders & $14(16.5 \%)$ \\
& \multicolumn{1}{c}{ Laboratory Characteristics } \\
\hline Hematological Disorders & $23(27.1 \%)$ \\
Anemia/ AIHA & $14(16.5 \%)$ \\
Leucopenia & $10(11.8 \%)$ \\
Lymphopenia & $22(25.9 \%)$ \\
Thrombocytopenia & \\
Immunological Disorders & \\
$\uparrow$ CRP & $44(51.8 \%)$ \\
$\downarrow$ C3 alone & $19(22.4 \%)$ \\
$\downarrow$ C4 alone & $11(13 \%)$ \\
$\downarrow$ C3 \& C4 both & $23(27.1 \%)$ \\
\hline
\end{tabular}

Table 2. Anti-Cardiolipin (ACA) and anti- $\beta 2$ glycoprotein $($ anti-- $\beta 2$ GP) antibodies in $(n=85)$.

\begin{tabular}{|c|c|c|}
\hline SLE type & $\begin{array}{l}\text { Anti-Cardiolipin } \\
\text { antibodies } \\
(\text { ACA) }(n=85)\end{array}$ & $\begin{array}{l}\text { Anti- } \beta 2 \text { glycoprotien } \\
\text { antibodies } \\
\text { (anti- } \beta 2 \text { GP) }(n=85)\end{array}$ \\
\hline \multicolumn{3}{|l|}{ Total SLE $(n=85)$} \\
\hline IgG positive & $11(12.9 \%)$ & $23(27.1 \%)$ \\
\hline IgM positive & $11(12.9 \%)$ & $27(31.8 \%)$ \\
\hline IgG + IgM positive & $3(3.5 \%)$ & $9(10.6 \%)$ \\
\hline IgG negative & $74(87.1 \%)$ & $62(72.9 \%)$ \\
\hline IgM negative & $74(87.1 \%)$ & $58(68.2 \%)$ \\
\hline \multicolumn{3}{|l|}{$\mathrm{LN}(n=32)$} \\
\hline IgG positive & $4(12.5 \%)$ & $8(25 \%)$ \\
\hline IgM positive & $4(12.5 \%)$ & $12(37.5 \%)$ \\
\hline IgG + IgM positive & $1(3.1 \%)$ & $3(9.4 \%)$ \\
\hline IgG negative & $28(87.5 \%)$ & $24(75 \%)$ \\
\hline IgM negative & $28(87.5 \%)$ & $20(62.5 \%)$ \\
\hline \multicolumn{3}{|l|}{ Non-LN $(n=53)$} \\
\hline IgG positive & $7(13.2 \%)$ & $15(28.3 \%)$ \\
\hline IgM positive & $7(13.2 \%)$ & $15(28.3 \%)$ \\
\hline IgG + IgM positive & $2(3.8 \%)$ & $6(11.3 \%)$ \\
\hline IgG negative & $46(86.8 \%)$ & $38(71.7 \%)$ \\
\hline IgM negative & $46(86.8 \%)$ & $38(71.7 \%)$ \\
\hline
\end{tabular}

IgG and IgM antibodies to ACA. When these patients were categorized further into LN and non-LN groups, ACA positivity for both IgG and IgM autoantibodies was slightly higher in non-LN group (13.2\%) as compared to $12.5 \%$ in $\mathrm{LN}$ group. Anti- $\beta 2$ GP positivity was $27.1 \%$ for IgG- $\beta 2$ GP and $31.8 \%$ for IgM- $\beta 2$ GP where as $10.6 \%$ of the patients developed anti- $\beta 2$ GP antibodies to both $\operatorname{IgG}$ and IgM subclasses. Among LN and non-LN groups,
IgG- $\beta 2$ GP positivity revealed a slightly higher incidence $(28.3 \%)$ in non-LN patients as compared to $\mathrm{LN}$ patients $(25 \%)$ where as LN patients showed a higher incidence for IgM- $\beta 2$ GP positivity ( $37.5 \%)$ as compared to $28.3 \%$ of patients in non-LN group. The cut off levels for IgG-ACA was $185 \mathrm{u} / \mathrm{ml}$, IgM-ACA is $186 \mathrm{u} / \mathrm{ml}$, IgG- $\beta 2$ GP is $156 \mathrm{u} / \mathrm{ml}$ and IgM- $\beta 2$ GP is $290 \mathrm{u} / \mathrm{ml}$ as per the normal individuals tested.

Table 3 gives the correlation of ACA and anti- $\beta 2$ GP levels with SLEDAI and other immunological parameters such as CRP, C3 and C4. IgM- $\beta 2$ GP positive LN patients had higher SLEDAI scores (mean \pm SD; $20 \pm$ $15.8)$ Out of 23 patients with IgG-anti- $\beta 2$ GP positivity, 15 (65.2\%) patients showed raised CRP levels where non-LN patients 11/15 (73.3\%) had higher CRP levels as compared to $4 / 8(50 \%)$ in LN group. Among IgM anti- $\beta 2$ GP positive patients $17 / 27$ patients $(63 \%)$ showed reduced $\mathrm{C} 3$ and $\mathrm{C} 4$ levels, where in LN group 10/12 patients (83.3\%) had reduced $\mathrm{C} 3$ and $\mathrm{C} 4$ levels as compared to $7 / 15$ patients (46.7\%) in non-LN group.

The distribution of clinical manifestations according to the ACR criteria among ACA and anti- $\beta 2$ GP positive patients at the time of evaluation was as shown in Table 4. ACA positive patients showed a higher incidence for clinical manifestations such as malar and discoid rash, photosensitivity, oral ulcers, vasculitis, alopecia, fever, arthritis and myositis. A slightly higher incidence for clinical manifestations such as renal disorders, serositis, neurological and hematological manifestations such as leucopenia, thrombocytopenia and autoimmune hemolytic anemia (AIHA) were noted among anti- $\beta 2$ GP patients. It was observed that among ACA positive patients, none of the patient had leucoepenia where as among anti- $\beta 2$ GP positive patients having leucopenia, WBC counts ranged between $(2.9-3.7) \times 10^{3} / \mu 1$ with mean \pm $\mathrm{SD}$ value of $3.3 \pm 0.6$. In a group of ACA positive patients having thrombocytopenia, platelet counts ranged between $(39-71) \times 10^{3} / \mu \mathrm{L}$ with a mean $\pm \mathrm{SD}$ value $5.5 \pm$ 22.6 where as anti- $\beta 2$ GP patients having thrombocytopenia showed platelet counts ranged between $(12-141)$ $\times 10^{3} / \mu 1$ with a slightly higher mean $\pm \mathrm{SD}$ value $(58.1 \pm$ 48.3). Table 5 shows distribution of clinical severity categorized into mild, moderate and severe based on the SLEDAI scores.

\section{Discussion}

Anti-phospholipid antibodies (APA) are a distinct group of autoantibodies that appear in a variety of autoimmune diseases, particularly Systemic Lupus Erythematosus (SLE). They are associated with clinical events such as arterial and/or venous thrombosis, and obstetric complications with a strong association of ACA with thrombosis, thrombocytopenia, recurrent fetal losses and Coombs' 
Table 3. Correlation of ACA and anti- $\beta 2$ GP levels with SLEDAI and other immunological parameters.

\begin{tabular}{|c|c|c|c|c|}
\hline \multirow{2}{*}{ SLE type } & \multicolumn{2}{|c|}{ Anti-Cardiolipin antibodies (ACA) } & \multicolumn{2}{|c|}{ Anti- $\beta 2$ glycoprotien antibodies (anti- $\beta 2$ GP) } \\
\hline & IgG & IgM & IgG & IgM \\
\hline Total SLE & 11 & 11 & 23 & 27 \\
\hline Range & $190-451$ & $186-1232$ & $156-410$ & $297-3000$ \\
\hline Mean + SD & $265.1 \pm 89.7$ & $392.9 \pm 299.7$ & $198.1 \pm 33.6$ & $847 \pm 683.2$ \\
\hline \multicolumn{5}{|l|}{ SLEDAI } \\
\hline Range & $4-43$ & $4-24$ & $4-35$ & $4-42$ \\
\hline Mean + SD & $14.6 \pm 11.1$ & $12.1 \pm 5.9$ & $14 \pm 9.1$ & $15.3 \pm 10.1$ \\
\hline$\uparrow \mathrm{CRP}$ & 11 & 9 & 15 & 14 \\
\hline$\downarrow \mathrm{C} 3$ alone & 3 & 0 & 3 & 0 \\
\hline$\downarrow$ C4 alone & 0 & 0 & 1 & 0 \\
\hline$\downarrow \mathrm{C} 3 \& \mathrm{C} 4$ both & 1 & 3 & 3 & 4 \\
\hline LN & 4 & 4 & 8 & 12 \\
\hline Range & $190-451$ & $186-459$ & $166-277$ & $338-3000$ \\
\hline Mean \pm SD & $261.3 \pm 126.7$ & $295.3 \pm 117.0$ & $212.3 \pm 42.4$ & $786.1 \pm 756.8$ \\
\hline \multicolumn{5}{|l|}{ SLEDAI } \\
\hline Range & $4-19$ & $4-15$ & $10-35$ & $8-64$ \\
\hline Mean \pm SD & $11.3 \pm 7.3$ & $10.8 \pm 5.0$ & $16.9 \pm 8.6$ & $20 \pm 15.8$ \\
\hline$\uparrow \mathrm{CRP}$ & 4 & 2 & 4 & 6 \\
\hline$\downarrow$ C3 alone & 0 & 0 & 1 & 0 \\
\hline$\downarrow \mathrm{C} 4$ alone & 0 & 0 & 1 & 0 \\
\hline$\downarrow \mathrm{C} 3 \& \mathrm{C} 4$ both & 0 & 1 & 1 & 2 \\
\hline Non-LN & 7 & 7 & 15 & 15 \\
\hline Range & $197-402$ & $210-1232$ & $156-410$ & $297-2150$ \\
\hline Mean \pm SD & $267.3 \pm 73.4$ & $448.7 \pm 364.5$ & $203.7 \pm 61.5$ & $895.3 \pm 641.2$ \\
\hline \multicolumn{5}{|l|}{ SLEDAI } \\
\hline Range & $4-43$ & $4-24$ & $4-43$ & $4-42$ \\
\hline Mean \pm SD & $16.6 \pm 12.9$ & $12.9 \pm 6.6$ & $12.9 \pm 9.3$ & $14.4 \pm 10.2$ \\
\hline$\uparrow \mathrm{CRP}$ & 7 & 7 & 11 & 8 \\
\hline$\downarrow \mathrm{C} 3$ alone & 3 & 0 & 2 & 0 \\
\hline$\downarrow \mathrm{C} 4$ alone & 0 & 0 & 0 & 0 \\
\hline$\downarrow \mathrm{C} 3 \& \mathrm{C} 4$ both & 1 & 2 & 2 & 2 \\
\hline
\end{tabular}

Table 4. Distribution of clinical manifestations according to ACR criteria among ACA and anti- $\beta 2$ GP positive patients.

\begin{tabular}{|c|c|c|}
\hline Clinical presentation & $\begin{array}{l}\text { ACA positives } \\
(\text { IgG and/or IgM) }(n=19)\end{array}$ & Anti- $\beta 2$ GP positives (IgG and/or IgM) $(n=41)$ \\
\hline $\begin{array}{l}\text { Malar Rash \&/ } \\
\text { Discoid Rash }\end{array}$ & $9(43.4 \%)$ & $14(34.1 \%)$ \\
\hline Photosensitivity & $4(21.1 \%)$ & $8(19.5 \%)$ \\
\hline Oral ulcers & $7(36.8 \%)$ & $11(26.8 \%)$ \\
\hline Arthritis & $14(73.7 \%)$ & $24(58.5 \%)$ \\
\hline Serositis & $6(31.6 \%)$ & $14(34.1 \%)$ \\
\hline Renal Disorders & $3(15.8 \%)$ & $8(19.5 \%)$ \\
\hline Neurological Disorders & $2(10.5 \%)$ & $8(19.5 \%)$ \\
\hline Hematological Disorders & $1(5.3 \%)$ & $5(12.1 \%)$ \\
\hline Systemic vascular thrombosis & $4(21.0 \%)$ & $10(24.4 \%)$ \\
\hline Myositis & $7(36.8 \%)$ & $10(24.4 \%)$ \\
\hline Alopecia & $8(42.1 \%)$ & $16(39.0 \%)$ \\
\hline Fever & $4(21.1 \%)$ & $7(17.1 \%)$ \\
\hline
\end{tabular}


Table 5. Distribution of clinical severity based on the SLEDAI scores.

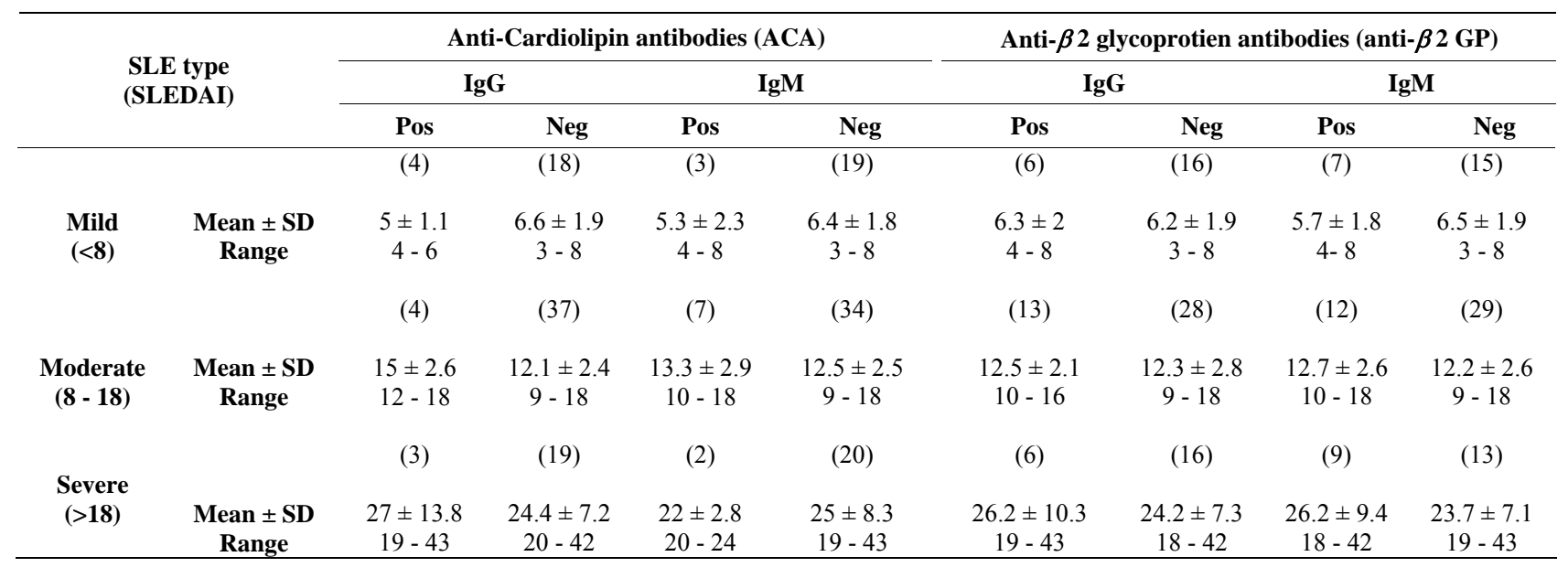

positivity in SLE and related autoimmune disorders [18-22].

Recently Mostafa et al., 2010 had reported an incidence of $16.7 \%$ for ACA among SLE patients [23]. Similar incidence was found in our study where IgG-ACA and IgM-ACA positivity was $12.9 \%$ each which was lower than anti- $\beta 2$ GP autoantibody positivity (IgG- $\beta 2$ GP: $27.1 \%$ and IgM- $\beta 2$ GP: $31.8 \%$ ). Other studies such as Petri et al., 2010 reported 47\% ACA and $32.5 \%$ anti- $\beta 2$ GP autoantibodies in SLE, Biggioggero et al., 2010 had reported $16.5 \%$ IgG-ACA and $9.4 \%$ of IgM-ACA and an incidence of $4.7 \%$ IgG- $\beta 2$ GP and $5.9 \%$ for IgM- $\beta 2$ GP antibodies, Jallouli et al., 2008 had reported 71.6\% for ACA and Descloux et al., 2008 had reported an incidence of $49 \%$ ACA [24,25,13,14]. In a study on South African SLE patients, Gould et al., 2006 had reported a very high incidence of $53 \%$ and $84 \%$ for ACA and anti- $\beta 2$ GP antibodies where as Al Arfaj et al., 2009 had reported an incidence of $49.7 \%$ and $33.5 \%$ for IgG-ACA and IgM-ACA respectively among Saudi Arabian SLE patients [26,27]. Recently Woo et al., 2010 had reported an incidence of $18.2 \%$ and $31.8 \%$ for IgG-ACA and IgM-ACA respectively and $5.7 \%$ for anti- $\beta 2$ GP to $\mathrm{IgG}$ and IgM isotypes in Korean SLE patients. Shrivastav et al., 2001 had reported 51\% IgG-ACA and 44.7\% IgG- $\beta 2$ GP autoantibodies [28,29].

Thrombosis varies in SLE patients from 7.2 to $12 \%$. Sarabi et al., 2005 reported that the most frequent causes of death in active SLE are infection and thrombosis [30]. The risk of thrombosis for SLE patients reported to be significantly higher and due to the increased incidence of traditional cardiovascular and nontraditional lupus-related thrombosis risk factors, SLE patients are at significantly increased risk of premature atherosclerosis and/or thrombosis. The prevalence of vascular events in SLE patients ranges between $10 \%$ and $30 \%$, for symptomatic coronary artery disease $6 \%$ - 20\%, stroke $2 \%-15 \%$, and subclinical coronary artery disease 30\% - 40\% [31]. Our study showed a higher incidence of systemic vascular thrombosis in anti- $\beta 2$ GP positive patients as compared to ACA positive patients with an equal distribution for venous and arterial thrombosis in both the groups.

Recurrent pregnancy loss (RPL) has been associated with APA including ACA and lupus anticoagulant. It had been reported that the risk of fetal loss is found to be increased in patients with hypertension, active SLE, LN, or abnormally low complement levels. Risk also increased for patients with APA: from $6 \%$ to $24 \%$ of patients with SLE are positive for LAC and $40 \%$ are positive for ACA [32-34]. In our study two patients had RPL and other two had $\mathrm{BOH}$, but they did not show the presence of ACA or anti- $\beta 2$ GP antibodies. Shrivastav et al., 2001 reported that incidence of neurological disorders such as seizures were noted in 9.4\% SLE patients which was significantly associated with the presence of ACA and anti- $\beta 2$ GP antibodies [29]. Neurological disorders were seen more in anti- $\beta 2$ GP positive patients than in ACA positive patients in our study.

Zheng $\mathrm{H}$ et al., 2009 reported that LN patients had elevated SLEDAI as compared to non-LN having elevated renal tissue injury, high serum creatinine, BUN and proteinuria levels with lower serum C3 levels [9]. Bhandari et al., 1998 had reported a significant reduction in C3 and C4 levels in ACA positive patients, with a strong relationship to disease activity/severity at presentation when compared with changes in ACA negative patients $(p<0.0 \%)$ where renal function at presentation was worse in patients with ACA positivity [35] It was suggested that ACA is a strong predictor for the presence of intra glomerular thrombi in patients with LN indicating the worse long term renal outcome in these patients. Our findings showed more complement consumption in 
anti- $\beta 2$ GP positive patients than ACA positives. Hence detection both ACA and anti- $\beta 2$ GP antibodies along with associated immune parameters were found to be helpful parameters to evaluate their possible association with disease severity in SLE patients. A long term follow up of patients having ACA and anti- $\beta 2$ GP antibodies without thrombotic event is required to detect their possible thrombotic event in future along with their clinical presentation.

\section{Acknowledgements}

We are grateful to Prof. Yehuda Shoenfeld, MD, Head, Department of Medicine at the Tel Aviv University, and Ramat Aviv, Israel for his inputs in correcting this manuscript and giving his valuable suggestions to modify it.

\section{REFERENCES}

[1] Y. Sherer, M. Blank and Y. Shoenfeld, “Anti-Phospholipid Syndrome (APS): Where Does It Come from?" Best Practice and Research Clinical Rehumatology, Vol. 21, No. 6, 2007, pp. 1071-1078. doi:10.1016/j.berh.2007.09.005

[2] E. M. Bevers, M. Galli, T. Barbui, et al., "Lupus Anticoagulant IgG's (LA) Are Not Directed to Phospholipids Only, but to a Complex of Lipid-Bound Human Prothrombin," Journal of Thrombosis and Haemostasis, Vol. 66, No. 6, 1991, pp. 629-632.

[3] S. Miyakis, M. D. Lockshin, T. Atsumi, D. W. Branch, R. L. Brey and R. Cervera, "International Consensus Statement on an Update of the Classification Criteria for Definite Antiphospholipid Syndrome (APS)," Journal of Thrombosis and Haemostasis, Vol. 4, No. 2, 2006, pp. 295-306. doi:10.1111/j.1538-7836.2006.01753.x

[4] H. P. McNeil, R. J. Simpson, C. N. Chesterman and S. A. Krilis, "Antiphospholipid Antibodies Are Directed against a Complex Antigen That Includes a Lipid-Binding Inhibitor of Coagulation: Beta 2-Glycoprotein I (Apolipoprotein H)," Proceedings of National Academy of Sciences of the United States of America, Vol. 87, No. 1, 1990, pp. 4120-4125. doi:10.1073/pnas.87.11.4120

[5] P. L. Meroni, N. D. Papa, D. Gambini, A. Tincami and G. Balesterari, "Antiphospholipid Antibodies and Endothelial Cells: An Unending Story," Lupus, Vol. 4, No. 3, 1995, pp. 169-171. doi:10.1177/096120339500400301

[6] D. S. Pisetsky, G. Gilkeson and E. W. St. Clair, "Systemic Lupus Erythematosus. Diagnosis and Treatment," Medical Clinics of North America, Vol. 81, No. 1, 1997, pp. 113-128. doi:10.1016/S0025-7125(05)70507-1

[7] G. S. Cooper, M. A. Dooley, E. L. Treadwell, E. W. St. Clair, C. G. Parks and G.S. Gilkeson, "Hormonal, Environmental, and Infectious Risk Factors for Developing Systemic Lupus Erythematosus," Arthritis and Rheumatism, Vol. 41, No. 10, 1998, pp. 1714-1724. doi:10.1002/1529-0131(199810)41:10<1714::AID-ART3 $>3.0 . \mathrm{CO} ; 2-\mathrm{U}$

[8] V. D. D’Agati and G. B Appel, "Lupus Nephritis: Pa- thology and Pathogenesis," In: D. J .Wallace and B. H. Hahn, Eds., Dubois' Lupus Erythematosus, $7^{\text {th }}$ Edition, Lippincott Williams \& Wilkins, Philadelphia, 2007, pp. 1094-1111.

[9] H. Zheng, Y. Chen, W. Ao, Y. Shen, X. W. Chen, M. Dai, X. D. Wang, Y. C. Yan and C. D. Yang, "Antiphospholipid Antibody Profiles in Lupus Nephritis with Glomerular Microthrombosis: A Prospective Study of 124 Cases," Arthritis Research and Therapy, Vol. 11, No. 3, 2009, p. R93. doi:10.1186/ar2736

[10] J. P. Grande, "Mechanisms of Progression of Renal Damage in Lupus Nephritis: Pathogenesis of Enal Scarring," Lupus, Vol. 7, No. 9, 1998, pp. 604-610. doi:10.1191/096120398678920721

[11] J. S. Levine, D. W. Branch and J. Rauch, "The Antiphospholipid Syndrome," The New England Journal of Medicine, Vol. 346, No. 2002, pp. 752-763. doi:10.1056/NEJMra002974

[12] M. L. Davies, S. P. Young, K. Welsh, M. Bunce, B. P. Wordsworth, et al., "Immune Responses to Native $\beta 2$-Glycoprotein I in Patients with Systemic Lupus Erythematosus and the Anti-Phospholipid Syndrome," Rheumatology, Vol. 41, No. 4, 2002, pp. 395-400. doi:10.1093/rheumatology/41.4.395

[13] M. Jallouli, M. Frigui, M. B. Hmida, S. Marzouk, N. Kaddour and Z. Bahloul, "Clinical and Immunological Manifestations of Systemic Lupus Erythematosus: Study on 146 South Tunisian Patients," Saudi Journal of Kidney Diseases and Transplantation, Vol. 19, No. 6, 2008, pp. 1001-1008.

[14] E. Descloux, I. Durieu, P. Cochat, D. V. Durand, J Ninet, N. Fabien and R. Cimaz, "Pediatric Systemic Lupus Erythematosus: Prognostic Impact of Antiphospholipid Antibodies," Rheumatology, Vol. 47, No. 2, 2008, pp. 183187. doi:10.1093/rheumatology/kem 335

[15] M. C. Hockberg, "Updating the American College of Rheumatology Revised Criteria for the Classification of Systemic Lupus Erythematosus," Arthritis and Rheumatism, Vol. 40, No. 9, 1997, p. 1725. doi:10.1002/art.1780400928

[16] C. Bombardier, F. F. Gladmsn, M. B. Urowit, D. Caron and C. H. Chang, "Derivation of SLEDAI: A Disease Activity Index for Lupus Patients. The Committee on Prognosis Studies in SLE," Arthritis and Rheumatism, Vol. 35, No. 6, 1995, pp. 630-640. doi:10.1002/art.1780350606

[17] J. J. Weening, V. D. Agati, M. M. Schwartz, S. V. Seshan, C. E. Alpers and G. B. Appel, "The Classification of Glomerulonephritis in Systemic Lupus Erythematosus Re visited," Journal of American Society of Nephrology, Vol. 15, No. 2, 2004, pp. 241-250. doi:10.1097/01.ASN.0000108969.21691.5D

[18] J. R. Mueh, K. D. Herbst and S. I. Rapaport, "Thrombosis in Patients with the Lupus Anticoagulant," Annals of Internal Medicine, Vol. 92, No. 2, 1980, pp. 156-159.

[19] L. O. Carreras, J. Vermylen, B. Spitz and A. V. Assche, "Lupus Anticoagulant and Inhibition of Prostacyclin Formation in Patients with Repeated Abortion, Intrauterine 
Growth Retardation and Intrauterine Death," British Journal of Obstetrics and Gynaecology, Vol. 88, No. 9, 1981, pp. 890-894. doi:10.1111/j.1471-0528.1981.tb02224.X

[20] M. L. Boey, C. B. Colaco, A. E. Gharavi, K. B. Elkon, S. Loizou and G. R. Hughes, "Thrombosis in Systemic Lupus Erythematosus: Striking Association with the Presence of Circulating Lupus Anticoagulant," British Medical Journal (Clinical Research Edition), Vol. 287, No. 6398, 1983, pp. 1021-1023.

[21] E. N. Harris, A. E. Gharavi and M. L. Boey, "Anti Cardiolipin Antibody Detection by Radioimmunoassay and Association with Thrombosis in Systemic Lupus Erythematosus," The Lancet, Vol. 332, No. 8361, 1983, pp. 1211-1214. doi:10.1016/S0140-6736(83)91267-9

[22] M. D. Lockshin and L. R. Sammaritano, "Lupus Pregnancy," Autoimmunity, Vol. 36, No. 1, 2011, pp. 33-40. doi:10.1080/0891693031000067313

[23] G. A. Mostafa, D. H. Ibrahim, A. A. Shehab and A. K. Mohammed, "The Role of Measurement of Serum Autoantibodies in Prediction of Pediatric Neuropsychiatric Systemic Lupus Erythematosus," Journal of Neuroimmunology, Vol. 227, No. 1-2, 2010, pp. 195-201.

[24] M. Petri, "Update on Anti-Phospholipid Antibodies in SLE: The Hopkins' Lupus Cohort," Lupus, Vol. 19, No. 4, 2010, pp. 419-423. doi:10.1177/0961203309360541

[25] M. Biggioggero and P. L. Meroni, "The Geoepidemiology of the Antiphospholipid Antibody Syndrome," Autoimmun Reviews, Vol. 9, No. 5, 2010, pp. A299-A304. doi:10.1016/j.autrev.2009.11.013

[26] T. Gould, M. Tikly, R. Asherson, S. Loizou and S. Singh, "Prevalence and Clinical Correlates of Anti-Phospholipid Antibodies in South Africans with Systemic Lupus Erythematosus," Scandinavian Journal of Rheumatology, Vol. 35, No. 1, 2006, pp. 29-34. doi:10.1080/03009740510026913

[27] A. S. Al Arfaj and N. Khalil, "Clinical and Immunological Manifestations in 624 SLE Patients in Saudi Arabia," Lupus, Vol. 18, No. 5, 2009, pp. 465-473.

\section{doi: $10.1177 / 0961203308100660$}

[28] K. S. Woo, K. E. Kim, J. M. Kim, J. Y. Han, W. T. Chung and K. H. Kim, "Prevalence and Clinical Associations of Lupus Anticoagulant, Anticardiolipin Antibodies, and Anti-Beta2-Glycoprotein I Antibodies in Patients with SLE," The Korean Journal of Laboratory Medicine, Vol. 30, No. 1, 2010, pp. 38-44. doi: $10.3343 / \mathrm{kj} / \mathrm{m} .2010 .30 .1 .38$

[29] A. Shrivastava, S. Dwivedi, A. Aggarwal and R. Misra, "Anti-Cardiolipin and Anti-Beta2 Glycoprotein I Antibodies in Indian Patients with Systemic Lupus Erythematosus: Association with the Presence of Seizures," $\mathrm{Lu}$ pus, Vol. 10, No. 1, 2001, pp. 45-50. doi:10.1191/096120301671577528

[30] Z. S. Sarabi, E. Chang, R. Bobba, D. Ibanez, D. Gladman, M. Urowitz, et al., "Incidence Rates of Arterial and Venous Thrombosis after Diagnosis and Systemic Lupus Erythematosus," Arthritis and Rheumatism, Vol. 53, No. 4, 2005, pp. 609-612. doi:10.1002/art.21314

[31] D. Erkan, "Lupus and Thrombosis," The Journal of Rheumatology, Vol. 32, No. 9, 2006, pp.1715-1717.

[32] M. Petri, M. Golbus, R. Anderson, Q. Whiting-O'Keefe, L. Corash and D. Hellmann, "Antinuclear Antibody, Lupus Anticoagulant, and Anticardiolipin Antibody in Women with Idiopathic Habitual Abortion. A Controlled Prospective Study of Forty-Four Women," Arthritis and Rheumatism, Vol. 30, No. 6, 1987, pp. 601-606. doi:10.1002/art.1780300601

[33] M. Petri and J. Allbritton, "Fetal Outcome of Lupus Pregnancy: A Retrospective Case-Control Study of the Hopkins Lupus Cohort," The Journal of Rheumatology, Vol. 20, No. 4, 1993, pp. 650-656.

[34] E. N. Harris, A. E. Gharavi and G. R. Hughes, "AntiPhospholipid Antibodies," Clinics in Rheumatic Diseases, Vol. 11, No. 3, 1985, pp. 591-609.

[35] S. Bhandari, P. Harnden, A. M. Brownjohn and J. H. Turney, "Association of Anti-Cardiolipin Antibodies with Intra-Glomerular Thrombi and Renal Dysfunction in Lupus Nephritis," QJM, Vol. 91, No. 6, 1998, pp. 401-409. 